This item was submitted to Loughborough's Research Repository by the author.

Items in Figshare are protected by copyright, with all rights reserved, unless otherwise indicated.

\title{
Verification theorems for models of optimal consumption and investment with annuitization
}

PLEASE CITE THE PUBLISHED VERSION

https://doi.org/10.1016/j.mathsocsci.2019.11.002

PUBLISHER

Elsevier BV

VERSION

AM (Accepted Manuscript)

\section{PUBLISHER STATEMENT}

This paper was accepted for publication in the journal Mathematical Social Sciences and the definitive published version is available at https://doi.org/10.1016/j.mathsocsci.2019.11.002.

LICENCE

CC BY-NC-ND 4.0

\section{REPOSITORY RECORD}

Park, Seyoung. 2019. "Verification Theorems for Models of Optimal Consumption and Investment with Annuitization". figshare. https://hdl.handle.net/2134/11274167.v1. 
Mathematical Social Sciences

Elsevier Editorial system(tm) for

Manuscript Draft

Manuscript Number: MSS-D-17-00320R2

Title: Verification Theorems for Optimal Consumption and Investment Problems with Annuitization

Article Type: Research Paper

Keywords: annuitization; existence; uniqueness; variational inequality

Corresponding Author: Dr. Seyoung Park, Ph.D.

Corresponding Author's Institution: Loughborough University

First Author: Seyoung Park, Ph.D.

Order of Authors: Seyoung Park, Ph.D.

Abstract: One can find it challenging to deal with verification theorems for optimal consumption and investment problems with annuitization. I pose a tractable framework to prove verification theorems for the problems. I revisit an annuitization model of Park (2015) and prove the verification theorem for the model. A key idea of the proof is the application of the variational inequality approach of Bensoussan and Lions (1982) to the annuitization problem solved by the suggested value function. Further, I obtain analytical comparative statics for the optimal consumption and investment strategies with annuitization. 


\section{Highlights}

- I prove the uniqueness and existence theorem for models of optimal consumption and investment with annuitization.

- I apply the variational inequality approach of Bensoussan and Lions (1982).

- I obtain analytic comparative statics for the optimal strategies. 


\title{
Verification Theorems for Models of Optimal Consumption and Investment with Annuitization
}

\author{
Seyoung Park \\ School of Business and Economics, Loughborough University, Epinal Way, Loughborough, Leicestershire, \\ UK, LE11 3TU, Tel:+44-7927-494518, E-mail: S.Park@lboro.ac.uk
}

\begin{abstract}
One can find it challenging to deal with verification theorems for optimal consumption and investment problems with annuitization. I pose a tractable framework to prove verification theorems for the problems. I revisit an annuitization model of Park (2015) and prove the verification theorem for the model. A key idea of the proof is the application of the variational inequality approach of Bensoussan and Lions (1982) to the annuitization problem solved by the suggested value function. Further, I obtain analytic comparative statics for the optimal consumption and investment strategies with annuitization.

Keywords: annuitization, existence, uniqueness, variational inequality

JEL classification: C61, G11
\end{abstract}

\section{Introduction}

Optimal life-cycle consumption and investment decisions with annuitization have received full attention in political economy, economics and finance spheres. Indeed, annuitization is arguably one of the most important life-cycle decisions just as consumption and investment in order to achieve successful retirement.

Proving verification theorems for models with annuitization can be a considerable chal- 
lenge because the question of existence of optimal strategies with discretionary stopping seems to be tricky to be answered. In addition, an expectation associated with annuitization is, in general, very difficult to compute. Compared to duality theory of Karatzas and Wang (2000), I pose a more tractable framework to prove verification theorems for problems with discretionary stopping, without resorting to the state price density (of the stochastic discount factor). Using the principle of dynamic programming, the proposed approach can be readily applied to proving verification theorems for problems with annuitization in a complete or an incomplete market.

I revisit an annuitization model of Park (2015) and prove the verification theorems for the model. While Yaari (1965) and Richard (1975) suggest full and immediate annuitization in the absence of bequest motives, i.e., all savings should be annuitized at all dates, ${ }^{1}$ this paper follows Park (2015) and thus, considers the case where individuals need to annuitize all of their wealth at one point in time. This resembles the current situation in the UK, where individuals determine when to start their retirement pension but must do so at one point in time. ${ }^{2}$ Actually, most variable annuity contracts in the US also provide individuals with an option to annuitize that can only be exercised once. My analysis would cover social security as well. In social security, benefits are provided in the form of a lifetime annuity based on a retirement age of 65 . If individuals can opt to retire earlier (as of age 62) or

\footnotetext{
${ }^{1}$ Davidoff et al. (2005) suggest much weaker sufficient conditions for such full and immediate annuitization.

${ }^{2}$ This is also akin to retirement annuity providing a stream of cash flows available for consumption during retirement years.
} 
later (up to age 70 ), they can withdraw a smaller or larger annuity, respectively. ${ }^{3}$ In this framework, I endogenously determine the optimal timing for full annuitization and this is effectively an optimal retirement problem. ${ }^{4}$

As a matter of fact, the date of annuitization does not need to be always the same with the date of retirement. In line with the recent trend of optimal claiming of life annuity income even after retirement in the US, on July 2014, the US Treasury department has allowed individuals to purchase a deferred income annuity (DIA) inside tax-sheltered retirement plans. Similar to life annuity considered in this paper, to purchase the DIA, individuals have to pay a lump sum at one distinct point in exchange for a defined (or a fixed) lifetime income. However, to purchase the DIA, individuals do not have to pay such a lump sum at only retirement. Rather, individuals can purchase it at a relatively young age and delay its income generating date until a relatively old age (usually after the normal

\footnotetext{
${ }^{3}$ Retirement age for individuals who was born before 1960 is 66 . For a detailed listing of retirement ages, please refer to www.socialsecurity.gov.

${ }^{4}$ There is a variety of distinct strands in the portfolio choice and retirement literature. This paper sits squarely within extensions of the retirement models proposed by Choi and Shim (2006), Farhi and Panageas (2007), Choi et al. (2008), Dybvig and Liu (2010), Jang et al. (2013), and Bensoussan et al. (2016). Within the optimal portfolio and retirement choice framework, Park (2015) shows that a nonlinear option-type element that is realistically present in the actual annuity market can induce voluntary and full annuitization. In particular, such annuitization decision can be regarded as an American-style option. Individuals would rather enjoy the extra leisure derived from their voluntary annuitization than the value of income obtained from their work, as soon as they touch a certain wealth threshold over which it is optimal to annuitize all of their wealth.
} 
retirement age). ${ }^{5}$ Although I do not take the DIA into this paper explicitly, in Section 5, I partially address the optimal claiming of life annuity income in the US by considering an exponentially distributed retirement age. In this case, individuals can withdraw their annuity income before or after the normal retirement age. Ultimately, I can investigate the effects of variation in retirement time on the annuitization decision.

One unique feature of the suggested framework is the application of the variational inequality approach of Bensoussan and Lions (1982) to the annuitization problem solved by the proposed value function. I verify the uniqueness and existence for the value function. Further, I obtain analytic comparative statics for the optimal strategies. In terms of tractable applications, I hope that this paper will lend itself to the verification for many other interesting utility maximization problems with discretionary stopping.

The paper is organized as follows. In Section 2, I specify the basic model in which the annuitization model of Park (2015) is revisited. In Section 3, I prove the uniqueness and existence theorem for the model. In Section 4, I provide analytica comparative statis for the derived optimal strategies. In Section 5, I consider more general case for annuitization than Section 2, where individuals are allowed to invest in both bonds and stocks after annuitization. In Section 6, I conclude the paper.

\footnotetext{
${ }^{5}$ The annuity income can be started to generate even after age 70 , but before age 85 . In this sense, such a DIA is also known as an Advanced Life Delayed Annuity.
} 


\section{The Basic Model}

In order to propose a tractable approach to proving verification theorems for optimal consumption and investment problems with annuitization, I need to solve a realistically calibrated model of annuitization. To allow for a realistic model of annuitization in the simplest possible setting in every other dimension, I revisit an annuitization model of Park (2015).

Let me specify the financial market. The market is comprised of two broad classes of assets: a bond (or a risk-free asset) and a stock (or a risky asset). The bond price $B_{t}$ is given by

$$
d B_{t}=r B_{t} d t
$$

where $r>0$ is the risk-free interest rate. The stock price $S_{t}$ follows a geometric Brownian motion:

$$
d S_{t}=\mu S_{t} d t+\sigma S_{t} d W_{t}
$$

where $\mu>r$ is the expected rate of the stock return, $\sigma>0$ is the stock volatility, and $W_{t}$ is a standard Brownian motion defined on a suitable probability space.

An individual receives a constant stream of labor income $I$ while she is working. I allow for the case where the individual can borrow against her labor income. That is, she can borrow up to the present value $I /(r+\nu)$ of labor income discounted at the sum of risk-free interest rate and mortality rate. Here, I assume that mortality rate is constant. I relax this assumption in Section 5. The individual is endowed with an amount of financial wealth 
$x>-I /(r+\nu) .{ }^{6}$ As long as she is working, the wealth dynamics follow

$$
d X_{t}=\left(r X_{t}-c_{t}+I\right) d t+\pi_{t} \sigma\left(d W_{t}+\theta d t\right), \quad X_{t}>-\frac{I}{r+\nu}, \quad 0 \leq t<\tau
$$

where $\pi$ is the dollar amount invested in the stock market and $\theta=(\mu-r) / \sigma$ denotes the Sharpe ratio.

As far as the model of Park (2015) is concerned, the individual aims to maximize the following life-time utility by optimally controlling her consumption $c$, investment $\pi$, and timing of voluntary annuitization $\tau$ :

$$
\Phi(x) \equiv \max _{(c, \pi, \tau)} E\left[\int_{0}^{\tau} e^{-(\beta+\nu) t} \frac{c_{t}^{1-\gamma}}{1-\gamma} d t+e^{-(\beta+\nu) \tau} \frac{\bar{\gamma}^{\gamma-\gamma^{*}}}{1-\gamma} \frac{\left(X_{\tau}(r+\nu)\right)^{1-\gamma}}{\beta+\nu}\right]
$$

where $E$ is the expectation taken at time $0, \beta>0$ is the individual's subjective discount rate, $\nu>0$ is the individual's constant mortality rate, $\gamma^{*}>0$ is the coefficient of relative risk aversion, and $\gamma \equiv 1-a\left(1-\gamma^{*}\right)>0$. Here, $0<a<1$ denotes a weight for consumption in the following Cobb-Douglas type utility preference:

$$
U\left(l_{t}, c_{t}\right)=\frac{1}{a} \frac{\left(l_{t}^{1-a} c_{t}^{a}\right)^{1-\gamma^{*}}}{1-\gamma^{*}},
$$

where $l_{t}$ is leisure at time $t$, which can be regarded as the time taken away from working. I consider two cases of leisure: (1) $l_{t}=\underline{l}$ while the individual is working $(2) l_{t}=\bar{l}(\bar{l}>\underline{l})$ when the individual annuitizes all of her wealth. I normalize $\underline{l}=1$. For the notational

\footnotetext{
${ }^{6}$ The implies that the individual can consume and invest in the stock as long as her financial wealth is above $-I /(r+\nu)$. If the wealth level approaches $-I /(r+\nu)$, then the individual cannot consume and invest any more. In this case, consumption and risky investment must be zero.
} 
convenience, I define

$$
K \equiv \frac{\bar{l}^{\gamma-\gamma^{*}}}{\beta+\nu}
$$

which represents the preference for leisure after voluntary annuitization. ${ }^{7}$ I assume that these is no bequest motive.

In my optimization problem (2), the optimal timing of voluntary annuitization is determined endogenously as the date at which the entire portfolio is geared toward riskless bonds, rather than a cross between stocks and bonds. This, in fact, reflects the canonical annuity results in Yaari (1965) and Richard (1975) that it is optimal to consume the entire annuity income in the absence of bequest motives. In line with this, I assume that at some future time $\tau$, individuals annuitize all of their wealth $X_{\tau}$ and consequently, consume at a rate of $X_{\tau}(r+\nu)$, which is the annual annuity income. ${ }^{8}$

\footnotetext{
${ }^{7}$ For instance, the preference for leisure stems from a disutility of work, household production, and cost savings. In reality, after annuitization the individual can have sufficient time to enjoy leisure such as shopping for bargains, preparing meals, and taking a cruise etc.

${ }^{8}$ There are two types of benefit plans for retirement. On the one hand, defined benefit plans guarantee a defined amount of income for remaining lifetimes after retirement, which are mainly considered in the paper. On the other hand, defined contribution plans (also better known as 401(k) retirement plans) provide a variable amount of income, depending on market/economy conditions. In light of the growing popularity of $401(\mathrm{k})$ retirement plans, annuities can likely to be indexed to stock returns. The current market seems to be coming around to the judgment in favor of defined contribution plans rather than defined benefit ones. To cover that situation as well, I investigate the case for which individuals can still have a great amount of flexibility after annuitization by allocating their financial resources to both bonds and stocks, instead of merely bonds. For the details, please refer to Section 5 .
} 


\section{Solutions}

For a fixed stopping time $\tau$, I define

$$
J_{\tau}(x) \equiv \max _{(c, \pi)} E\left[\int_{0}^{\tau} e^{-(\beta+\nu) t} \frac{c_{t}^{1-\gamma}}{1-\gamma} d t+e^{-(\beta+\nu) \tau} K \frac{\left(X_{\tau}(r+\nu)\right)^{1-\gamma}}{1-\gamma}\right]
$$

Then the value function $\Phi(x)$ given by (2) can be rewritten by

$$
\Phi(x)=\max _{\tau} J_{\tau}(x)
$$

This is the proposed annuitization problem. I combine the variational inequality approach of Bensoussan and Lions (1982) with an analysis of the problem that I can solve in closed form. Following Bensoussan and Lions (1982), the associated variational inequalities are

$$
\left\{\begin{array}{l}
(\beta+\nu) \Phi(x) \geq \max _{(c, \pi)}\left\{\frac{c^{1-\gamma}}{1-\gamma}+(r x-c+I+\pi \sigma \theta) \Phi^{\prime}(x)+\frac{1}{2} \pi^{2} \sigma^{2} \Phi^{\prime \prime}(x)\right\} \\
\Phi(x) \geq K \frac{(x(r+\nu))^{1-\gamma}}{1-\gamma}, \\
{\left[(\beta+\nu) \Phi(x)-\max _{(c, \pi)}\left\{\frac{c^{1-\gamma}}{1-\gamma}+(r x-c+I+\pi \sigma \theta) \Phi^{\prime}(x)+\frac{1}{2} \pi^{2} \sigma^{2} \Phi^{\prime \prime}(x)\right\}\right]} \\
\times\left[\Phi(x)-K \frac{(x(r+\nu))^{1-\gamma}}{1-\gamma}\right]=0,
\end{array}\right.
$$

for any $x>-I /(r+\nu)$. By using the following optimality conditions (or the first order conditions)

$$
c_{t}^{*}=\Phi^{\prime}(x)^{-1 / \gamma} \text { and } \pi_{t}^{*}=-\frac{\theta}{\sigma} \frac{\Phi^{\prime}(x)}{\Phi^{\prime \prime}(x)}
$$


the variational inequalities formulated by (3) are rewritten as

$$
\left\{\begin{array}{l}
(\beta+\nu) \Phi(x) \geq(r x+I) \Phi^{\prime}(x)-\frac{\theta^{2}}{2} \frac{\Phi^{\prime}(x)^{2}}{\Phi^{\prime \prime}(x)}+\frac{\gamma}{1-\gamma} \Phi^{\prime}(x)^{1-1 / \gamma} \\
\Phi(x) \geq K \frac{(x(r+\nu))^{1-\gamma}}{1-\gamma}, \\
{\left[(\beta+\nu) \Phi(x)-\left\{(r x+I) \Phi^{\prime}(x)-\frac{\theta^{2}}{2} \frac{\Phi^{\prime}(x)^{2}}{\Phi^{\prime \prime}(x)}+\frac{\gamma}{1-\gamma} \Phi^{\prime}(x)^{1-1 / \gamma}\right\}\right]} \\
\times\left[\Phi(x)-K \frac{(x(r+\nu))^{1-\gamma}}{1-\gamma}\right]=0
\end{array}\right.
$$

for any $x>-I /(r+\nu)$.

It seems that it is almost impossible to derive an explicit solution of (5). In addition, the general question of the uniqueness and existence of the solution can be tricky. I first verify the uniqueness for the value function $\Phi$ that solves (5).

Theorem 3.1. (Uniqueness) There exists an unique solution $\Phi(x)$ that satisfies the variational inequalities given by (5) such that it is $C^{1}$, piecewise $C^{2}$, and

$$
\Phi^{\prime}(x)>0 \text { and } \Phi^{\prime \prime}(x)<0
$$

for any $x>-I /(r+\nu)$.

Proof. A solution of (5) such that it is $C^{1}$, piecewise $C^{2}$, and it satisfies (6) for any $x>-I /(r+\nu)$ is necessarily unique. To prove this, I consider the set $\Gamma$ of upper solutions of (5) that satisfies the first and second inequalities except for the last equality in (5). I assume that the set $\Gamma$ is not empty. I want to show that if an element of $\Gamma$ satisfies the last equality in (5), then it is smaller than any other element of $\Gamma$ for any $x>-I /(r+\nu)$. The solution of (5) is consequently unique. 
Let $\phi$ be a solution of (5). Then it is an element of $\Gamma$ and it also satisfies the last equality in (5). Note that as long as

$$
\phi(x)>K \frac{(x(r+\nu))^{1-\gamma}}{1-\gamma},
$$

the inequalities in (5) are satisfied for $\phi$.

For any $x_{0}$ such that

$$
\phi\left(x_{0}\right)>K \frac{\left(x_{0}(r+\nu)\right)^{1-\gamma}}{1-\gamma},
$$

consider the largest interval $(\underline{b}, \bar{b})$ containing $x_{0}$ for which the strict inequality in (5) holds. That is, if $\bar{b}>0, \underline{b}<\infty$, then

$$
\phi(\underline{b})=K \frac{(\underline{b}(r+\nu))^{1-\gamma}}{1-\gamma}, \quad \phi(\bar{b})=K \frac{(\bar{b}(r+\nu))^{1-\gamma}}{1-\gamma},
$$

and

$$
(\beta+\nu) \phi(x)=(r x+I) \phi^{\prime}(x)-\frac{\theta^{2}}{2} \frac{\phi^{\prime}(x)^{2}}{\phi^{\prime \prime}(x)}+\frac{\gamma}{1-\gamma} \phi^{\prime}(x)^{1-1 / \gamma}, \quad \underline{b}<x<\bar{b} .
$$

It follows that $\phi^{\prime \prime}(x)$ is continuous on the open interval $(\underline{b}, \bar{b})$. Because $\phi^{\prime \prime}(x)<0$, I can also write

$$
\begin{aligned}
(\beta+\nu) \phi(x) & =\max _{(c, \pi)}\left\{\frac{c^{1-\gamma}}{1-\gamma}+(r x-c+I+\pi \sigma \theta) \phi^{\prime}(x)+\frac{1}{2} \pi^{2} \sigma^{2} \phi^{\prime \prime}(x)\right\} \\
& =\frac{\left(c^{*}\right)^{1-\gamma}}{1-\gamma}+\left(r x-c^{*}+I+\pi^{*} \sigma \theta\right) \phi^{\prime}(x)+\frac{1}{2}\left(\pi^{*}\right)^{2} \sigma^{2} \phi^{\prime \prime}(x),
\end{aligned}
$$

where $c^{*}, \pi^{*}$ achieve the maximum.

Consider an element $\widetilde{\phi}$ of $\Gamma$. Then I can get

$$
\begin{gathered}
(\beta+\nu) \widetilde{\phi}(x) \geq \frac{\left(c^{*}\right)^{1-\gamma}}{1-\gamma}+\left(r x-c^{*}+I+\pi^{*} \sigma \theta\right) \widetilde{\phi}^{\prime}(x)+\frac{1}{2}\left(\pi^{*}\right)^{2} \sigma^{2} \widetilde{\phi}^{\prime \prime}(x) \text { a.e. } x \in(\underline{b}, \bar{b}), \\
\widetilde{\phi}(\underline{b}) \geq \phi(\underline{b}), \quad \widetilde{\phi}(\bar{b}) \geq \phi(\bar{b}),
\end{gathered}
$$


and

$(\beta+\nu)(\widetilde{\phi}(x)-\phi(x)) \geq\left(r x-c^{*}+I+\pi^{*} \sigma \theta\right)\left(\widetilde{\phi}^{\prime}(x)-\phi^{\prime}(x)\right)+\frac{1}{2}\left(\pi^{*}\right)^{2} \sigma^{2}\left(\widetilde{\phi}^{\prime \prime}(x)-\phi^{\prime \prime}(x)\right)$.

By the maximum principle (Friedman, 1982), I can deduce

$$
\widetilde{\phi}(x) \geq \phi(x) \quad \text { on } \quad[\underline{b}, \bar{b}]
$$

Further, for any $x>-I /(r+\nu)$ such that

$$
\phi(x)=K \frac{(x(r+\nu))^{1-\gamma}}{1-\gamma}
$$

the following relationship holds:

$$
\widetilde{\phi}(x) \geq \phi(x)
$$

Therefore,

$$
\widetilde{\phi}(x) \geq \phi(x)
$$

for any $x>-I /(r+\nu)$. Hence, $\phi$ becomes a minimum element of $\Gamma$, which proves the uniqueness of a solution to (5). Q.E.D.

Having established the uniqueness of solution, I conjecture that the problem can be solved by finding an optimal stopping boundary $\bar{x}$, which should be determined by the appropriate value-matching and smooth-pasting conditions. Then the variational inequalities 
(5) can be restated as follows:

$$
\left\{\begin{array}{l}
(\beta+\nu) \Phi(x)=(r x+I) \Phi^{\prime}(x)-\frac{\theta^{2}}{2} \frac{\Phi^{\prime}(x)^{2}}{\Phi^{\prime \prime}(x)}+\frac{\gamma}{1-\gamma} \Phi^{\prime}(x)^{1-1 / \gamma}, \quad-I /(r+\nu)<x<\bar{x} \\
\Phi(x)=K \frac{(x(r+\nu))^{1-\gamma}}{1-\gamma}, \quad x \geq \bar{x} \\
\Phi(\bar{x})=K \frac{(\bar{x}(r+\nu))^{1-\gamma}}{1-\gamma}, \\
\Phi^{\prime}(\bar{x})=K(\bar{x}(x+\nu))^{-\gamma} .
\end{array}\right.
$$

In the following theorem, I verify the existence for the value function $\Phi$ solves (5). There are two steps associated with proving the existence theorem. Firstly, I derive in closed-form the value function $\Phi$ that solves (7). Secondly, I show that the function $\Phi$ also satisfies the variational inequalities in (5).

Theorem 3.2. (Existence) I assume that ${ }^{9}$

$$
\begin{aligned}
& \gamma>1 \\
& (r+\nu)<K^{1 / \gamma} \eta<\left\{\frac{\gamma \eta}{\gamma-1}+\frac{\theta^{2}}{2}\left(\alpha-\frac{1}{\gamma}\right)\right\} /\left\{\frac{(\beta+\nu)}{\gamma-1}+\left(r+\frac{\theta^{2} \alpha}{2}\right) \frac{1}{r+\nu}\right\} \\
& \frac{1}{r+\nu}>1 .
\end{aligned}
$$

Then the solution $\Phi(x)$ of (5) satisfies

$$
\Phi^{\prime}(x)>0 \quad \text { and } \quad \Phi^{\prime \prime}(x)<0,
$$

\footnotetext{
${ }^{9} \mathrm{I}$ assume that $\gamma>1$, which is widely adopted in the existing literature and consistent with the data. Note that $K$ representing the preference for leisure after annuitization determines the amount of consumption jump when the individual enters annuitization. Specifically,$$
\frac{c_{\tau^{+}}^{*}}{c_{\tau_{-}}^{*}}=\bar{l}^{\left(\gamma-\gamma^{*}\right) / \gamma}=K^{1 / \gamma}(\beta+\nu)
$$

Therefore, the second condition involving $K^{1 / \gamma}$ implies that the individual needs to appropriately counteract the discrete change in leisure from $\underline{l}$ to $\bar{l}$ at the date of annuitization, giving rise to the manageable size of consumption jump. The last condition always holds with reasonable parameter values of $r$ and $\nu$.
} 
and has the following closed form:

$$
\Phi(x)=\left\{\begin{array}{l}
\frac{1}{\beta+\nu}\left[\left\{\left(r+\frac{\theta^{2}}{2 \gamma}\right) \frac{1}{\eta}+\frac{\gamma}{1-\gamma}\right\} \lambda(x)^{1-1 / \gamma}+\left(r+\frac{\theta^{2} \alpha}{2}\right) A \lambda(x)^{1-\alpha}\right], \quad-\frac{I}{r+\nu}<x<\bar{x}, \\
K \frac{(x(r+\nu))^{1-\gamma}}{1-\gamma}, \quad x \geq \bar{x}
\end{array}\right.
$$

where

$$
\lambda(x)=\Phi^{\prime}(x),
$$

$\lambda(x)$ is a decreasing function in $x$ and has an implicit relationship with $x$ as the following:

$$
G(\lambda(x)) \equiv \frac{1}{\eta} \lambda(x)^{-1 / \gamma}+A \lambda(x)^{-\alpha}=x+\frac{I}{r}, \quad-\frac{I}{r+\nu}<x<\bar{x},
$$

and

$$
\begin{aligned}
& \alpha=\frac{\left(\beta+\nu-r+\frac{1}{2} \theta^{2}\right)+\sqrt{\left(\beta+\nu-r+\frac{1}{2} \theta^{2}\right)^{2}+2 \theta^{2} r}}{\theta^{2}}>1, \\
& \eta=\frac{\gamma-1}{\gamma}\left(r+\frac{\theta^{2}}{2 \gamma}\right)+\frac{\beta+\nu}{\gamma}>0, \\
& A=\left[\left(\frac{K^{1 / \gamma}}{r+\nu}-\frac{1}{\eta}\right) K^{-1 / \gamma} \bar{x}(r+\nu)+\frac{I}{r}\right] K^{\alpha}(\bar{x}(r+\nu))^{-\gamma \alpha}>0, \\
& \bar{x}=K^{1 / \gamma}\left(1+\frac{\theta^{2} \alpha}{2 r}\right)\left(\frac{I}{r+\nu}\right) /\left[K^{1 / \gamma}\left\{\frac{(\beta+\nu)}{1-\gamma}-\left(r+\frac{\theta^{2} \alpha}{2}\right) \frac{1}{r+\nu}\right\}-\frac{\gamma}{1-\gamma}+\frac{\theta^{2}}{2 \eta}\left(\alpha-\frac{1}{\gamma}\right)\right]>0 .
\end{aligned}
$$

Proof. I prove the theorem in three steps.

Step 1. I prove that the function $\Phi(x)$ given by (10) solves the optimal stopping problem formulated by (7). I can directly compute that

$$
\begin{aligned}
\Phi^{\prime}(x) & =\frac{1}{\beta+\nu}\left[\left\{\left(r+\frac{\theta^{2}}{2 \gamma}\right) \frac{1}{\eta}+\frac{\gamma}{1-\gamma}\right\}\left(1-\frac{1}{\gamma}\right) \lambda(x)^{-1 / \gamma} \lambda^{\prime}(x)+\left(r+\frac{\theta^{2} \alpha}{2}\right) A(1-\alpha) \lambda(x)^{-\alpha} \lambda^{\prime}(x)\right] \\
& =\frac{1}{\beta+\nu}\left[-\frac{1}{\eta}\left(\frac{\beta+\nu}{\gamma}\right) \lambda(x)^{-1 / \gamma}-(\beta+\nu) \alpha A \lambda(x)^{-\alpha}\right] \lambda^{\prime}(x) \\
& =\left[-\frac{1}{\gamma \eta} \lambda(x)^{-1 / \gamma}-\alpha A \lambda(x)^{-\alpha}\right] \lambda^{\prime}(x), \quad-\frac{I}{r+\nu}<x<\bar{x},
\end{aligned}
$$

where the second equality results from the following relationship:

$$
-\frac{1}{2} \theta^{2} \alpha(\alpha-1)+(\beta+\nu-r) \alpha+r=0 .
$$


I also can get

$$
G^{\prime}(\lambda(x)) \equiv \frac{\partial G(\lambda(x))}{\partial \lambda(x)}=-\frac{1}{\gamma \eta} \lambda(x)^{-1-1 / \gamma}-\alpha A \lambda(x)^{-1-\alpha}
$$

from (11). Then,

$$
\Phi^{\prime}(x)=\lambda(x) G^{\prime}(\lambda(x)) \lambda^{\prime}(x)=\lambda(x), \quad-\frac{I}{r+\nu}<x<\bar{x},
$$

because from (11) when I differentiate the equation

$$
G(\lambda(x))=x+\frac{I}{r}
$$

with respect to $x$, I obtain

$$
G^{\prime}(\lambda(x)) \lambda^{\prime}(x)=1, \quad-\frac{I}{r+\nu}<x<\bar{x} .
$$

From (13),

$$
G^{\prime \prime}(\lambda(x))\left(\lambda^{\prime}(x)\right)^{2}+G^{\prime}(\lambda(x)) \lambda^{\prime \prime}(x)=0, \quad-\frac{I}{r+\nu}<x<\bar{x} .
$$

From (12), I deduce immediately

$$
\Phi^{\prime \prime}(x)=\lambda^{\prime}(x), \quad-\frac{I}{r+\nu}<x<\bar{x} .
$$

Thus, the function $\Phi(x)$ given by (10) can be rewritten as

$$
\Phi(x)=\frac{1}{\beta+\nu}\left[r G(\lambda(x)) \lambda(x)-\frac{\theta^{2}}{2} \lambda(x)^{2} G^{\prime}(\lambda(x))+\frac{\gamma}{1-\gamma} \lambda(x)^{1-1 / \gamma}\right]
$$

for any $-I /(r+\nu)<x<\bar{x}$. Accordingly, the equations (12), (13), (14), and (15) yield

$$
(\beta+\nu) \Phi(x)=(r x+I) \Phi^{\prime}(x)-\frac{\theta^{2}}{2} \frac{\Phi^{\prime}(x)^{2}}{\Phi^{\prime \prime}(x)}+\frac{\gamma}{1-\gamma} \Phi^{\prime}(x)^{1-1 / \gamma}, \quad-I /(r+\nu)<x<\bar{x},
$$


which proves that the function $\Phi(x)$ given by (10) satisfies the first equation in $(7)$.

Now I want to show that the function $\Phi(x)$ given by (10) satisfies the value-matching and smooth-pasting conditions:

$$
\begin{aligned}
& \Phi(\bar{x})=K \frac{(\bar{x}(r+\nu))^{1-\gamma}}{1-\gamma} \\
& \Phi^{\prime}(\bar{x})=K(\bar{x}(x+\nu))^{-\gamma}
\end{aligned}
$$

The smooth-pasting condition must hold if I define a dual variable $\underline{\lambda}$ of the free boundary $\bar{x}$ as follows:

$$
\underline{\lambda} \equiv \lambda(\bar{x})=\Phi^{\prime}(\bar{x})=K(\bar{x}(r+\nu))^{-\gamma}
$$

where I have used the relationship of (12). By computing the value of $\Phi(x)$ for $-I /(r+\nu)<$ $x<\bar{x}$ at $x=\bar{x}$, I get from (16) that

$$
\begin{aligned}
\Phi(\bar{x}) & =\frac{1}{\beta+\nu}\left[\left\{\left(r+\frac{\theta^{2}}{2 \gamma}\right) \frac{1}{\eta}+\frac{\gamma}{1-\gamma}\right\} \lambda(\bar{x})^{1-1 / \gamma}+\left(r+\frac{\theta^{2} \alpha}{2}\right) A \lambda(\bar{x})^{1-\alpha}\right] \\
& =\frac{1}{\beta+\nu}\left[\left\{\left(r+\frac{\theta^{2}}{2 \gamma}\right) \frac{1}{\eta}+\frac{\gamma}{1-\gamma}\right\} \underline{\lambda}^{1-1 / \gamma}+\left(r+\frac{\theta^{2} \alpha}{2}\right) A \underline{\lambda}^{1-\alpha}\right] .
\end{aligned}
$$

Note that from (11) and (17),

$$
G(\underline{\lambda})=\bar{x}+\frac{I}{r}=\frac{K^{1 / \gamma} \underline{\lambda}^{-1 / \gamma}}{r+\nu}+\frac{I}{r}
$$

From (11), I know that

$$
G(\underline{\lambda})=\frac{1}{\eta} \underline{\lambda}^{-1 / \gamma}+A \underline{\lambda}^{-\alpha}
$$

accordingly,

$$
\frac{1}{\eta} \underline{\lambda}^{-1 / \gamma}+A \underline{\lambda}^{-\alpha}=\frac{K^{1 / \gamma} \underline{\lambda}^{-1 / \gamma}}{r+\nu}+\frac{I}{r}
$$

Thus, I can rewrite the value of $\Phi(x)$ for $-I /(r+\nu)<x<\bar{x}$ at $x=\bar{x}$ as

$$
\Phi(\bar{x})=\frac{1}{\beta+\nu}\left[\left\{\left(r+\frac{\theta^{2} \alpha}{2} \frac{K^{1 / \gamma}}{r+\nu}\right)+\frac{\gamma}{1-\gamma}+\frac{\theta^{2}}{2 \eta}\left(\frac{1}{\gamma}-\alpha\right)\right\} \underline{\lambda}^{1-1 / \gamma}+\left(1+\frac{\theta^{2} \alpha}{2 r}\right) I \underline{\lambda}\right] .
$$


Then, the value-matching condition

$$
\Phi(\bar{x})=K \frac{(\bar{x}(r+\nu))^{1-\gamma}}{1-\gamma}
$$

is equivalent to

$$
\underline{\lambda}^{-1 / \gamma}=\left(1+\frac{\theta^{2} \alpha}{2 r}\right) I /\left[K^{1 / \gamma}\left\{\frac{(\beta+\nu)}{1-\gamma}-\left(r+\frac{\theta^{2} \alpha}{2}\right) \frac{1}{r+\nu}\right\}-\frac{\gamma}{1-\gamma}+\frac{\theta^{2}}{2 \eta}\left(\alpha-\frac{1}{\gamma}\right)\right]
$$

by using the definition of $\underline{\lambda}$ given in (17). Note that from the definition (17) of the dual variable $\underline{\lambda}$, I deduce immediately

$\bar{x}=K^{1 / \gamma}\left(1+\frac{\theta^{2} \alpha}{2 r}\right)\left(\frac{I}{r+\nu}\right) /\left[K^{1 / \gamma}\left\{\frac{(\beta+\nu)}{1-\gamma}-\left(r+\frac{\theta^{2} \alpha}{2}\right) \frac{1}{r+\nu}\right\}-\frac{\gamma}{1-\gamma}+\frac{\theta^{2}}{2 \eta}\left(\alpha-\frac{1}{\gamma}\right)\right]>0$

and

$$
\bar{x}>0
$$

which results from the assumption (8).

Step 2. I would like to prove that the function $\Phi(x)$ given by (10) satisfies variational inequalities in (5). It is enough to show that the first inequality in (5) holds for $x>\bar{x}$ and the second one in (5) holds for $-I /(r+\nu)<x<\bar{x}$.

Consider the second case where $-I /(r+\nu)<x<\bar{x}$. I define

$$
\Psi(x) \equiv \Phi(x)-K \frac{(x(r+\nu))^{1-\gamma}}{1-\gamma}
$$

I know

$$
\Psi(\bar{x})=0
$$

and

$$
\Psi^{\prime}(x)=\Phi^{\prime}(x)-K(x(r+\nu))^{-\gamma}=\lambda(x)-K(x(r+\nu))^{-\gamma} .
$$


Then I want to show that

$$
\Psi^{\prime}(x)<0
$$

which is equivalent to

$$
\frac{\lambda(x)^{-1 / \gamma} K^{1 / \gamma}}{r+\nu}>x
$$

To show this, I firstly verify that

$$
\lambda^{\prime}(x)<0
$$

which is equivalent to

$$
G^{\prime}(\lambda(x))<0
$$

due to the relationship of (13). I know that the function $G$ given by

$$
G(\lambda(x))=\frac{1}{\eta} \lambda(x)^{-1 / \gamma}+A \lambda(x)^{-\alpha}
$$

is convex in $\lambda(x)$ because $-1 / \gamma<0, A$ is positive, and $-\alpha<-1$. Further,

$$
G(\underline{\lambda})=\frac{1}{\eta} \underline{\lambda}^{-1 / \gamma}+A \underline{\lambda}^{-\alpha}<+\infty \quad \text { and } \quad G(\infty)=0
$$

as a result, the function $G$ is monotonic decreasing i.e.,

$$
G^{\prime}(\lambda(x))<0
$$

Hence, the case of $-I /(r+\nu)<\bar{x}$ is equivalent to $\lambda(x)>\underline{\lambda}$. Now I want to show that

$$
G(\lambda) \leq \frac{K^{1 / \gamma} \underline{\lambda}^{-1 / \gamma}}{r+\nu}+\frac{I}{r}
$$

for any $\lambda>\underline{\lambda}$. I define

$$
V(\lambda) \equiv G(\lambda)-\frac{K^{1 / \gamma} \lambda^{-1 / \gamma}}{r+\nu}-\frac{I}{r}
$$


then

$$
V(\underline{\lambda})=0 \quad \text { and } \quad V(\infty)=-\frac{I}{r}
$$

Note that the function $G$ satisfies the following differential solution:

$$
-\frac{1}{2} \theta^{2} \lambda^{2} G^{\prime \prime}(\lambda)-\left(\theta^{2}+\beta+\nu-r\right) \lambda G^{\prime}(\lambda)+r G(\lambda)=\lambda^{-1 / \gamma}
$$

Thus,

$$
\begin{aligned}
-\frac{1}{2} \theta^{2} \lambda^{2} V^{\prime \prime}(\lambda)-\left(\theta^{2}+\beta+\nu-r\right) \lambda V^{\prime}(\lambda)+r V(\lambda) & =\lambda^{-1 / \gamma}-\frac{K^{1 / \gamma} \eta \lambda^{-1 / \gamma}}{r+\nu}-I \\
& =\left(1-\frac{K^{1 / \gamma} \eta}{r+\nu}\right) \lambda^{-1 / \gamma}-I \\
& <0,
\end{aligned}
$$

where the last inequality comes from the assumption (8) in the theorem. By the maximum principle (Friedman, 1982), I can show that $V(\lambda)<0$ for any $\lambda>\underline{\lambda}$ and hence, show that the second inequality in (5) folds for $-I /(r+\nu)<x<\bar{x}$.

Now I turn to the case where $x>\bar{x}$. In this case, I have

$$
\Phi(x)=K \frac{(x(r+\nu))^{1-\gamma}}{1-\gamma} .
$$

By substituting this into the first inequality in (5), I obtain

$$
x \geq I /\left[\left\{\frac{(\beta+\nu)}{1-\gamma}-\frac{\theta^{2}}{2 \gamma}-\frac{\gamma}{1-\gamma} K^{-1 / \gamma}\right\}(r+\nu)-r\right] .
$$

Finally,

$$
\bar{x}>I /\left[\left\{\frac{(\beta+\nu)}{1-\gamma}-\frac{\theta^{2}}{2 \gamma}-\frac{\gamma}{1-\gamma} K^{-1 / \gamma}\right\}(r+\nu)-r\right]
$$

must hold if I assume that

$$
\frac{1}{r+\nu}>1
$$


which is the assumption given in the theorem.

Step 3. I want to prove that the solution $\Phi(x)$ given by (10) satisfies

$$
\Phi^{\prime}(x)>0 \quad \text { and } \quad \Phi^{\prime \prime}(x)<0
$$

For the case where $x \geq \bar{x}$, I can easily deduce that

$$
\Phi^{\prime}(x)=K(x(r+\nu))^{-\gamma}>0 \quad \text { and } \quad \Phi^{\prime \prime}(x)=-\gamma K(x(r+\nu))^{-\gamma-1}<0 .
$$

Now I consider the case of $-I /(r+\nu)<x<\bar{x}$. In this case, from (12) I know that

$$
\Phi^{\prime}(x)=\lambda(x)
$$

Note that from (19),

$$
\Phi^{\prime \prime}(x)=\lambda^{\prime}(x)<0
$$

which implies that $\lambda(x)$ is a monotonic decreasing function in $x$. Accordingly, from (17)

$$
\Phi^{\prime}(x)=\lambda(x)>\lambda(\bar{x})=\underline{\lambda}=K(\bar{x}(r+\nu))^{-\gamma}>0 . \quad \text { Q.E.D. }
$$

\section{Analytic Comparative Statics}

I have proved the verification theorems consisting of the uniqueness and existence for the value function $\Phi$. Next, I provide analytic comparative statics for the optimal consumption and investment strategies with annuitization.

Corollary 4.1. Optimal consumption and risky investment policies for the individual with voluntary annuitzation are given by

$$
\begin{aligned}
c^{*} & =\eta\left(x+\frac{I}{r}-A \lambda(x)^{-\alpha}\right), \\
\pi^{*} & =\frac{\theta}{\gamma \sigma}\left(x+\frac{I}{r}\right)+\frac{\theta}{\sigma}\left(\alpha-\frac{1}{\gamma}\right) A \lambda(x)^{-\alpha} .
\end{aligned}
$$


Proof. According to the optimality conditions given by (4),

$$
\begin{aligned}
c^{*} & =\Phi^{\prime}(x)^{-1 / \gamma} \\
& =\lambda(x)^{-1 / \gamma} \\
& =\eta\left(x+\frac{I}{r}-A \lambda(x)^{-\alpha}\right),
\end{aligned}
$$

where the last equality results from the rearrangement of (11). Further,

$$
\begin{aligned}
\pi^{*} & =-\frac{\theta}{\sigma} \frac{\Phi^{\prime}(x)}{\Phi^{\prime \prime}(x)} \\
& =-\frac{\theta}{\sigma} \lambda(x) G^{\prime}(\lambda(x)) \\
& =\frac{\theta}{\gamma \sigma \eta} \lambda(x)^{-1 / \gamma}+\frac{\theta \alpha}{\sigma} A \lambda(x)^{-\alpha} \\
& =\frac{\theta}{\gamma \sigma}\left(x+\frac{I}{r}\right)+\frac{\theta}{\sigma}\left(\alpha-\frac{1}{\gamma}\right) A \lambda(x)^{-\alpha} . \quad \text { Q.E.D. }
\end{aligned}
$$

My optimal consumption plan generalizes the permanent-income hypothesis (PIH) of Friedman (1957). The PIH is the annuity value of total wealth consisting of the sum of financial wealth $x$ and present value $I / r$ of future labor income discounted at risk-free interest rate. It is formulated by

$$
c=\eta\left(x+\frac{I}{r}\right), \quad \eta=\frac{\gamma-1}{\gamma}\left(r+\frac{\theta^{2}}{2 \gamma}\right)+\frac{\beta+\nu}{\gamma},
$$

which implies that consumption is higher as the Sharpe ratio $\theta$ is higher, the subjective discount rate $\beta$ higher, the mortality rate $\nu$ higher, or the risk aversion coefficient $\gamma$ lower. The optimal consumption strategy for the individual with voluntary annuitization in the corollary is determined by the smaller annuity value of total wealth, as I compared to the case in the absence of annuitization. Specifically, the annuity value decreases from $x+I / r$ 
to $x+I / r-A \lambda(x)^{-\alpha}$. The smaller annuity value induces the individual to accumulate more wealth to enter voluntary annuitization as soon as possible. Further, the effect of annuitization becomes noticeable as the individual's wealth approaches the wealth threshold $\bar{x}$, because $\lambda(x)$ is decreasing in wealth $x$.

The optimal risky investment policy also generalizes the traditional investment rule proposed by Merton (1969). The conventional investment wisdom is given as follows. It is optimal to invest more in the risky asset as the Sharpe ratio increases or risk aversion decreases. This is given by

$$
\pi=\frac{\theta}{\gamma \sigma}\left(x+\frac{I}{r}\right) .
$$

In the companion paper, Park (2015) provides the economic intuition that voluntary annuitization can be interpreted as exercising an American-type option. My analytic comparative statics shows that the option positively affects the individual's optimal investment in the risky asset. The rational behind this is straightforward: for individuals who want to annuitize all of their wealth, wealth accumulation is more attractive and hence investment in the stock market is more aggressive, ceteris paribus.

\section{General Cases for Annuitization}

In this section, I consider optimal consumption and investment problems with annuitization in a more general setting in three ways. Firstly, at the date of voluntary annuitization, the entire portfolio is allocated to only bonds, rather than a mix of bonds and stocks. Given a great amount of freedom (until recently) to individuals even after annuitization when determining how to save and invest for retirement years, I allow for a general case 
for which they can invest both in bonds and in stocks with a defined amount of annuity income.

Secondly, the date of voluntary annuitization has been the same with the time of retirement. I relax this assumption. In addition to annuitization decision, I allow an individual to retire based on a retirement age of 65 . The retirement date is assumed to follow an exponential distribution with positive intensity. ${ }^{10}$

Finally, I relax the assumption that mortality rate is constant. One can allow for a highly nonlinear hazard into death by introducing an additional stochastic process for time-varying $\nu_{s}$.

\footnotetext{
${ }^{10}$ Choosing both a retirement date and an annuitization date would be a considerable challenge, because one should determine two free boundaries (or two wealth thresholds) for both retirement and annuitization. For example, I might add one more dimension for annuitization by considering a style of barrier control of Milevsky and Young (2007) and Huang et al. (2017). Milevsky and Young (2007) allow retirees to annuitize any fraction of their wealth at any time. Huang et al. (2017) consider the optimal purchasing and claiming of deferred income annuities through which individuals can purchase annuities at a relatively young age and delay their income generating date until a relatively old age (usually after the normal retirement age). However, these studies are limited to consider both retirement and annuitization. To the best of my knowledge, it will be a first attempt to consider retirement and annuitization at the same time by using both an optimal stopping and a barrier control. I leave this to future research as an open problem. Instead, I consider an exponentially distributed retirement age just as mortality event. Then the annuitization date is, at least, no longer the same with the retirement time.
} 


\subsection{Saving and Investing for Retirement Years}

The wealth dynamics follow with $X_{0}=x$ : before annuitization,

$$
d X_{t}=\left(r X_{t}-c_{t}+I\right) d t+\pi_{t} \sigma\left(d W_{t}+\theta d t\right), \quad X_{t}>-\frac{I}{r+\nu}, \quad 0 \leq t<\tau
$$

after annuitization,

$$
d X_{t}=\left(r X_{t}-c_{t}+I_{2}\right) d t+\pi_{t} \sigma\left(d W_{t}+\theta d t\right), \quad X_{t}>-\frac{I_{2}}{r+\nu}, \quad t \geq \tau,
$$

where $I_{2}\left(I_{2}<I\right)$ is a specified amount of annuity income for lifetimes.

I address the general case for annuitization in the two steps. One is to solve the intertemporal optimal consumption and investment problem after annuitization. The other is to solve the annuitization problem with the derived value function after annuitization.

The optimal consumption and portfolio choice problem after annuitization is given by

$$
\tilde{\Phi}(x) \equiv \max _{(c, \pi)} E\left[\int_{0}^{\infty} e^{-(\beta+\nu) t} l^{(1-a)\left(1-\gamma^{*}\right)} \frac{c_{t}^{1-\gamma}}{1-\gamma} d t\right]
$$

subject to the wealth dynamics (20) after annuitization. Following Merton (1969), the Hamilton-Jacobi-Bellman (HJB) equation is

$$
(\beta+\nu) \tilde{\Phi}(x)=\max _{(c, \pi)}\left\{\bar{l}^{(1-a)\left(1-\gamma^{*}\right)} \frac{c^{1-\gamma}}{1-\gamma}+\left(r x-c+I_{2}+\pi \sigma \theta\right) \tilde{\Phi}^{\prime}(x)+\frac{1}{2} \pi^{2} \sigma^{2} \tilde{\Phi}^{\prime \prime}(x)\right\}
$$

for any $x>-I_{2} /(r+\nu)$. The optimality conditions (or the first order conditions) for consumption $c$ and investment $\pi$ are

$$
c_{t}^{*}=\bar{l}^{(1-a)\left(1-\gamma^{*}\right) / \gamma} \tilde{\Phi}^{\prime}(x)^{-1 / \gamma} \text { and } \pi_{t}^{*}=-\frac{\theta}{\sigma} \frac{\tilde{\Phi}^{\prime}(x)}{\tilde{\Phi}^{\prime \prime}(x)} .
$$


After substituting these for $c$ and $\pi$ in (21), I obtain the following nonlinear differential equation:

$$
(\beta+\nu) \tilde{\Phi}(x)=\left(r x+I_{2}\right) \tilde{\Phi}^{\prime}(x)-\frac{\theta^{2}}{2} \frac{\tilde{\Phi}^{\prime}(x)^{2}}{\tilde{\Phi}^{\prime \prime}(x)}+\frac{\gamma}{1-\gamma} \bar{l}^{(1-a)(1-\gamma *) / \gamma} \tilde{\Phi}^{\prime}(x)^{1-1 / \gamma}
$$

Next, I derive in closed-form the solution to the above equation as follows:

$$
\tilde{\Phi}(x)=\tilde{K} \frac{\left(x+I_{2} / r\right)^{1-\gamma}}{1-\gamma}
$$

where

$$
\tilde{K}=\bar{l}^{(1-a)\left(1-\gamma^{*}\right)}\left(\frac{1}{\eta}\right)^{\gamma}
$$

Having solve the optimal consumption and investment problem after annuitization, let me get back to the annuitization problem. By the principle of dynamic programming, the annuitization problem is to maximize the following life-time utility by optimally controlling per-period consumption $c$, investment $\pi$, and timing of voluntary annuitization $\tau$ :

$$
\begin{aligned}
\Phi(x) & \equiv \max _{(c, \pi, \tau)} E\left[\int_{0}^{\tau} e^{-(\beta+\nu) t} \frac{c_{t}^{1-\gamma}}{1-\gamma} d t+e^{-(\beta+\nu) \tau} \int_{\tau}^{\infty} e^{-(\beta+\nu)(t-\tau)} \bar{l}^{(1-a)\left(1-\gamma^{*}\right)} \frac{c_{t}^{1-\gamma}}{1-\gamma} d t\right] \\
& =\max _{(c, \pi, \tau)} E\left[\int_{0}^{\tau} e^{-(\beta+\nu) t} \frac{c_{t}^{1-\gamma}}{1-\gamma} d t+e^{-(\beta+\nu) \tau} \tilde{\Phi}\left(X_{\tau}\right)\right] \\
& =\max _{(c, \pi, \tau)} E\left[\int_{0}^{\tau} e^{-(\beta+\nu) t} \frac{c_{t}^{1-\gamma}}{1-\gamma} d t+e^{-(\beta+\nu) \tau} \tilde{K} \frac{\left(X_{\tau}+I_{2} / r\right)^{1-\gamma}}{1-\gamma}\right] .
\end{aligned}
$$

Similar to the case for which only bonds are considered after annuitization, I derive the associated variational inequalities just as (5):

$$
\left\{\begin{array}{l}
(\beta+\nu) \Phi(x) \geq(r x+I) \Phi^{\prime}(x)-\frac{\theta^{2}}{2} \frac{\Phi^{\prime}(x)^{2}}{\Phi^{\prime \prime}(x)}+\frac{\gamma}{1-\gamma} \Phi^{\prime}(x)^{1-1 / \gamma} \\
\Phi(x) \geq \tilde{K} \frac{\left(x+I_{2} / r\right)^{1-\gamma}}{1-\gamma}, \\
{\left[(\beta+\nu) \Phi(x)-\left\{(r x+I) \Phi^{\prime}(x)-\frac{\theta^{2}}{2} \frac{\Phi^{\prime}(x)^{2}}{\Phi^{\prime \prime}(x)}+\frac{\gamma}{1-\gamma} \Phi^{\prime}(x)^{1-1 / \gamma}\right\}\right]} \\
\times\left[\Phi(x)-\tilde{K} \frac{\left(x+I_{2} / r\right)^{1-\gamma}}{1-\gamma}\right]=0
\end{array}\right.
$$


for any $x>-I /(r+\nu)$. Theorem 3.1 can be applied to (22) as the only difference comes from the value function $\tilde{K}\left(x+I_{2} / r\right)^{1-\gamma} /(1-\gamma)$ instead of $K(x(r+\nu))^{1-\gamma} /(1-\gamma)$ after annuitization. Then the variational inequalities (22) can be rewritten by the following optimal stopping problem with a free boundary $\bar{x}$ :

$$
\left\{\begin{array}{l}
(\beta+\nu) \Phi(x)=(r x+I) \Phi^{\prime}(x)-\frac{\theta^{2}}{2} \frac{\Phi^{\prime}(x)^{2}}{\Phi^{\prime \prime}(x)}+\frac{\gamma}{1-\gamma} \Phi^{\prime}(x)^{1-1 / \gamma}, \quad-I /(r+\nu)<x<\bar{x}, \\
\Phi(x)=\tilde{K} \frac{\left(x+I_{2} / r\right)^{1-\gamma}}{1-\gamma}, \quad x \geq \bar{x}, \\
\Phi(\bar{x})=\tilde{K} \frac{\left(\bar{x}+I_{2} / r\right)^{1-\gamma}}{1-\gamma}, \\
\Phi^{\prime}(\bar{x})=\tilde{K}\left(\bar{x}+I_{2}\right)^{-\gamma} .
\end{array}\right.
$$

Given that the optimal stopping problem constructed above is the same as in (7) except for the value function after annuitization, Theorem 3.2 also can be applied to (23) using the similar argument.

\subsection{Annuitization with Earlier or Later Retirement}

Retirement age for individuals who was born before 1960 was 66. I can consider variation in this retirement age by assuming that an individual's retirement age is exponentially distributed with positive intensity $\delta$. Specifically,

$$
P\left[\tau^{\delta}>t\right]=e^{-\delta t}
$$

where $\tau^{\delta}$ is the retirement age. Given this specification, the expected time to retirement is $1 / \delta$. If we consider an individual who just starts off her permanent job at the age of 30 , then she is expected to retire at 65 when $\delta=1 / 35$. In the range of $\underline{\delta}<\delta=1 / 35<\bar{\delta}$, the individual can retire earlier with $\bar{\delta}$ or later with $\underline{\delta}$ than with $\delta=1 / 35$. 
Two future scenarios are possible. If the individual claims her annuity income earlier than her expected retirement age of 65 with $\delta=\bar{\delta}$, then she is expected to withdraw a smaller annuity. If the individual claims her annuity income later than her expected retirement age of 65 with $\delta=\underline{\delta}$, then she is expected to withdraw a larger annuity. More specifically, the individual aims to maximize the following life-time utility by optimally controlling her consumption $c$, investment $\pi$, and timing of voluntary annuitization $\tau$ :

$$
\Phi(x) \equiv \max _{(c, \pi, \tau)} E\left[\int_{0}^{\tau} e^{-(\beta+\nu+\delta) t} \frac{c_{t}^{1-\gamma}}{1-\gamma} d t+e^{-(\beta+\nu+\delta) \tau} \frac{\bar{l}^{\gamma-\gamma^{*}}}{1-\gamma} \frac{\left(X_{\tau}(r+\nu)\right)^{1-\gamma}}{\beta+\nu+\delta}\right]
$$

where the last term on the right hand side decreases as $\delta$ increases. That is, in terms of the utility value of annuity income, the individual enjoys less utility with earlier retirement when $\delta=\bar{\delta}$ than with later retirement when $\delta=\underline{\delta}$.

Given the similarity between (2) and (24) except for the last term, Theorem 3.1 and Theorem 3.2 can be applied to (24) as well.

\subsection{Time-Varying Mortality Rate}

I introduce the following time-varying mortality rate for $\nu_{s}:{ }^{11}$ with $\nu_{0}=\nu>0$,

$$
d \nu_{s}=\nu_{s}\left(\mu_{s}^{\nu} d s+\sigma_{s}^{\nu} d W_{s}^{*}\right)
$$

\footnotetext{
${ }^{11}$ In this case, however, highly nonlinear hazard process $\nu$ is likely to violate the condition that$$
\text { probability of }\{\tau \leq t\}=\int_{0}^{t} \nu_{s} d s \leq 1 \text {, }
$$

where $\tau$ is distributed according to an exponential distribution with time-varying $\nu$. This is caused by the random drift in the stochastic process of $\nu$. I leave this issue to future research as an interesting extension. Instead, I confirm that the condition is satisfied with a wide range of parameter values of $\mu_{s}^{\nu}$ and $\sigma_{s}^{\nu}$.
} 
where $\mu_{s}^{\nu}$ and $\sigma_{s}^{\nu}$ are functions with respect to time variable $s$ and $W_{s}^{*}$ is a standard onedimensional Brownian motion having an instantaneous correlation $\rho \in[-1,1]$ with $W_{s}$ in the stock price process, i.e.,

$$
d W_{s} \cdot d W_{s}^{*}=\rho d s
$$

For simplicity, I impose the following conditions for the parameters of $\mu_{s}^{\nu}$ and $\sigma_{s}^{\nu}$ :

$$
\mu_{s}^{\nu}=-\nu<0 \text { and } \sigma_{s}^{\nu}=\sigma^{\nu}>0
$$

These conditions do make sense in terms of economic plausibility when studying the effects of variation in mortality rate. Our time-varying mortality rate reduces to the constant mortality rate when $\sigma^{\nu}=0$ :

$$
d \nu_{s}=-\nu \nu_{s} d s
$$

or equivalently,

$$
\nu_{s}=\nu e^{-\nu s}
$$

as a result,

$$
P\left[\tau_{M}>t\right]=\int_{t}^{\infty} \nu e^{-\nu s} d s=e^{-\nu t}
$$

where $\tau_{M}$ is the date of mortality. Then our time-varying mortality rate can represent fluctuation in mortality rate when $\sigma^{\nu}>0$.

In the presence of time-varying mortality rate, the present value of $\$ 1$ discounted at 
the risk-free interest rate $r$ is

$$
\begin{aligned}
& E\left[\int_{0}^{\tau_{M}} e^{-r t} d t\right] \\
& =E\left[\int_{0}^{\infty} \nu e^{-\nu s} e^{\sigma^{\nu} W_{s}^{*}-\frac{1}{2}\left(\sigma^{\nu}\right)^{2} s} \int_{0}^{s} e^{-r t} d t d s\right] \\
& =E\left[\int_{0}^{\infty} e^{-r t} \int_{t}^{\infty} \nu e^{-\nu s} e^{\sigma^{\nu} W_{s}^{*}-\frac{1}{2}\left(\sigma^{\nu}\right)^{2} s} e^{-r t} d s d t\right] \\
& =E^{Q}\left[\int_{0}^{\infty} e^{-r t} \int_{t}^{\infty} \nu e^{-\nu s} d s d t\right] \\
& =E^{Q}\left[\int_{0}^{\infty} e^{-(r+\nu) t} d t\right] \\
& =\frac{1}{r+\nu}
\end{aligned}
$$

where $Q$ is the new measure that has the following relation with the physical measure $P$ :

$$
P^{Q}(A) \equiv \int_{A} e^{\sigma^{\nu} W_{t}^{*}(\omega)-\frac{1}{2}\left(\sigma^{\nu}\right)^{2} t(\omega)} d P(\omega)
$$

Then when the individuals enters full annuitization at time $t$ with an amount of wealth $\$ X_{t}$, she is expected to receive annuity income $\$ X_{t}(r+\nu)$ per year continuously.

With time-varying mortality rate $\nu_{s}$, an individual aims to maximize the following life-time utility by optimally controlling her consumption $c$, investment $\pi$, and timing of 
voluntary annuitization $\tau$ in the new $Q$ measure instead of the physical $P$ measure:

$$
\begin{aligned}
\Phi(x) & \equiv \max _{(c, \pi, \tau)} E\left[\int_{0}^{\tau \wedge \tau_{M}} e^{-\beta t} \frac{c_{t}^{1-\gamma}}{1-\gamma} d t+e^{-\beta\left(\tau \wedge \tau_{M}\right)} \frac{\bar{l}^{\gamma-\gamma^{*}}}{1-\gamma} \frac{\left(X_{\tau \wedge \tau_{M}}(r+\nu)\right)^{1-\gamma}}{\beta+\nu}\right] \\
& =\max _{(c, \pi, \tau)} E\left[\int_{0}^{\tau} \nu e^{-\nu s} e^{\sigma^{\nu} W_{s}^{*}-\frac{1}{2}\left(\sigma^{\nu}\right)^{2} s} \int_{0}^{s} 0 d t d s+\int_{\tau}^{\infty} \nu e^{-\nu s} e^{\sigma^{\nu} W_{s}^{*}-\frac{1}{2}\left(\sigma^{\nu}\right)^{2} s} \int_{0}^{\tau} e^{-\beta t} \frac{c_{t}^{1-\gamma}}{1-\gamma} d t d s\right. \\
& \left.+\int_{0}^{\tau} \nu e^{-\nu s} e^{\sigma^{\nu} W_{s}^{*}-\frac{1}{2}\left(\sigma^{\nu}\right)^{2} s} e^{-\beta s} 0 d s+\int_{\tau}^{\infty} \nu e^{-\nu s} e^{\sigma^{\nu} W_{s}^{*}-\frac{1}{2}\left(\sigma^{\nu}\right)^{2} s} e^{-\beta \tau} \frac{\bar{l}^{\gamma-\gamma^{*}}}{1-\gamma} \frac{\left(X_{\tau}(r+\nu)\right)^{1-\gamma}}{1-\gamma} d s\right] \\
& =\max _{(c, \pi, \tau)} E\left[\int_{0}^{\tau} e^{-\beta t} \frac{c_{t}^{1-\gamma}}{1-\gamma} \int_{\tau}^{\infty} \nu e^{-\nu s} e^{\sigma^{\nu} W_{s}^{*}-\frac{1}{2}\left(\sigma^{\nu}\right)^{2} s} d s d t\right. \\
& \left.+\int_{\tau}^{\infty} e^{-\beta \tau} \frac{\bar{l}^{\gamma-\gamma^{*}}}{1-\gamma} \frac{\left(X_{\tau}(r+\nu)\right)^{1-\gamma}}{1-\gamma} \nu e^{-\nu s} e^{\sigma^{\nu} W_{s}^{*}-\frac{1}{2}\left(\sigma^{\nu}\right)^{2} s} d s\right] \\
& =\max _{(c, \pi, \tau)} E^{Q}\left[\int_{0}^{\tau} e^{-\beta t} \frac{c_{t}^{1-\gamma}}{1-\gamma} \int_{\tau}^{\infty} \nu e^{-\nu s} d s d t+e^{-\beta \tau} \frac{\bar{l}^{\gamma-\gamma^{*}}}{1-\gamma} \frac{\left(X_{\tau}(r+\nu)\right)^{1-\gamma}}{1-\gamma} \int_{\tau}^{\infty} \nu e^{-\nu s} d s\right] \\
& =\max _{(c, \pi, \tau)} E^{Q}\left[\int_{0}^{\tau} e^{-(\beta+\nu) t} \frac{c_{t}^{1-\gamma}}{1-\gamma} d t+e^{-(\beta+\nu) \tau} \frac{\bar{l}^{\gamma-\gamma^{*}}}{1-\gamma} \frac{\left(X_{\tau}(r+\nu)\right)^{1-\gamma}}{\beta+\nu}\right] .
\end{aligned}
$$

If we define

$$
W_{t}^{Q} \equiv W_{t}-\rho \sigma^{\nu} t
$$

then with Girsanov Theorem $W_{t}^{Q}$ becomes a standard Brownian motion under $Q$. Hence, the individual's wealth dynamics evolve as under $Q$

$$
d X_{t}=\left(r X_{t}-c_{t}+I\right) d t+\pi_{t} \sigma\left(d W_{t}^{Q}+\left(\theta+\rho \sigma^{\nu}\right) d t\right), \quad X_{t}>-\frac{I}{r+\nu}, \quad 0 \leq t<\tau
$$

Comparing to the wealth dynamics given in (1), it seems that the Sharpe ratio $\theta$ is replaced by $\theta+\rho \sigma^{\nu}$.

With the replaced Sharpe ratio, Theorem 3.1 and Theorem 3.2 can be applied to (25) using the similar argument. 


\section{Conclusion}

I have verified the theorems with focuses on the uniqueness and existence for the value function of intertemporal optimal consumption and investment problem with annuitization. The methodological advantage of the constructed verification theorems can be readily applied to the verification for many other interesting utility maximization problems with optimal stopping in a complete or an incomplete market, not resorting to the state price density (or the stochastic discount factor).

By no means I do claim that I address all the issues that are likely to be relevant for actual annuitization decisions (e.g., bequest motive, time-varying mortality hazard, etc.). Rather, I view the model developed in this paper as a complement to our current understanding of more realistic, typically numerically solved, models of annuitization. Many interesting extensions to this model should be relatively tractable.

Important extensions would be to include characteristics that are realistically present in actual annuitization plans with retirement. In this paper, the date of annuitization is assumed to be the date of retirement, enjoying more leisure after annuitization (or retirement). However, this is not necessarily true. A more realistic model would allow an open market structure for which individuals can annuitize any fraction of their wealth at any time rather than at one distinct point in time (e.g., retirement).

In light of the growing popularity of defined contribution pension plans instead of defined benefit pension plans, annuities could be indexed to stock returns and thus, should respond to shocks in the stock market. The risk associated with annuities would impact 
the conventional results on annuitization (e.g., Yaari, 1965; Richard, 1975) stating that all savings should be annuitized in the absence of bequest motive.

The other extension would be to allow mortality rate to be time-varying. The constant mortality hazard assumption is obviously limited to address practical issues associated with annuitization. Rather than the constant mortality rate, one can adopt a widely used Gompertz force of mortality, which is common in the actuarial literature for annuity pricing. Additional randomness in the nonlinear hazard process would alter the quantitative features of the current model. 


\section{References}

[1] Bensoussan, A., Lions, J. L. 1982. Applications of Variational Inequalities in Stochastic Control. North Holland.

[2] Bensoussan, A., Jang, B. G., Park, S. 2016. Unemployment Risks and Optimal Retirement in an Incomplete Market. Operations Research. 64, 1015-1032.

[3] Choi, K. J., Shim, G. 2006. Disutility, Optimal Retirement, and Portfolio Selection. Mathematical Finance. 16, 443-467.

[4] Choi, K. J., Shim, G., Shin, Y. H. 2008. Optimal Portfolio, Consumption-Leisure and Retirement Choice Problem with CES Utility. Mathematical Finance. 18, 445-472.

[5] Davidoff, T., Brown, J. R., Diamond, P. A. 2005. Annuities and Individual Welfare. American Economic Review. 95, 1573-1590.

[6] Dybvig, P. H., Liu, H. 2010. Lifetime Consumption and Investment: Retirement and Constrained Borrowing. Journal of Economic Theory. 145, 885-907.

[7] Dybvig, P. H., Liu, H. 2011. Verification Theorems for Models of Optimal Consumption and Investment with Retirement and Constrained Borrowing. Mathematics of Operations Research. 36, 620-635.

[8] Farhi, E., Panageas, S. 2007. Saving and Investing for Early Retirement: A Theoretical Analysis. Journal of Financial Economics. 83, 87-121. 
[9] Friedman, A. 1982. Variational Principles and Free-Boundary Problems. Wiley, New York.

[10] Friedman, M. 1957. A Theory of the Consumption Function. Princeton: Princeton University Press.

[11] Huang, H., Milevsky, M. A., Young, V. R. 2017. Optimal Purchasing of Deferred Income Annuities When Payout Yields are Mean-Reverting. Review of Finance. 327-361.

[12] Jang, B. G., Park, S., Rhee, Y. 2013. Optimal Retirement with Unemployment Risks. Journal of Banking and Finance. 37, 3585-3604.

[13] Karatzas, I., Wang, H. 2000. Utility Maximization with Discretionary Stopping. SIAM Journal on Control and Optimization. 39, 306-329.

[14] Merton, R. C. 1969. Lifetime Portfolio Selection under Uncertainty: The ContinuousTime Case. Review of Economics and Statistics. 51, 247-257.

[15] Milevsky, M. A., Young, V. R. 2007. Annuitization and Asset Allocation. Journal of Economic Dynamics \& Control. 31, 3138-3177.

[16] Park, S. 2015. A Generalization of Yaari's Result on Annuitization with Optimal Retirement. Economics Letters. 137, 17-20.

[17] Richard, S. 1975. Optimal Consumption, Portfolio and Life Insurance Rules for an 
Uncertain Lived Individual in a Continuous Time Model. Journal of Financial Economics.

2, 187-203.

[18] Yaari, E. 1965. Uncertain Lifetime, Life Insurance, and the Theory of the Consumer.

Review of Economics Studies. 32, 137-150.

\section{References}

